\title{
Deliciosos polimorfos
}

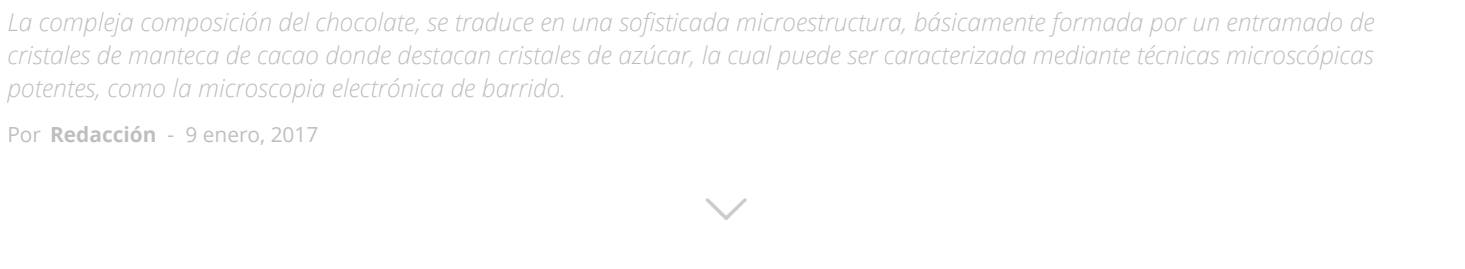

El polimorfismo, tan habitual y conocido por geólogos en minerales como la calcita-aragonito, el diamante-grafito y el grupo de la sílice, se extiende a muchos otros campos. Desde la cristalografía y la mineralogía, dando un gran salto en los materiales pero no en los fenómenos, el polimorfismo también tiene lugar en productos alimentarios. En particular, los triacilgliceroles, los cuales constituyen los componentes mayoritarios de grasas y aceites, tales como el chocolate, el aceite de oliva o el jamón ibérico, muestran un complejo comportamiento polimórfico (Larsson et al., 2006). La caracterización de sus formas cristalinas resulta crucial en aplicaciones de la industria alimentaria para satisfacer las necesidades del consumidor, optimizar procesos industriales o desarrollar nuevos productos. Sin embargo, el polimorfismo también podría ser utilizado como herramienta discriminatoria entre diferentes categorías de un mismo producto alimentario, así como para la detección de acciones fraudulentas (Bayés-García et al., 2016).

De este modo, la industria alimentaria debe hacer frente a unas expectativas cada vez más exigentes por parte del consumidor. En el caso del chocolate, el producto se puede presentar en formatos muy diversos, pero en todos los casos, su aspecto siempre debe ser atractivo y tentador. El brillo y la fina textura característica de este producto alimentario se deben a la selección de la forma polimórfica adecuada, que aporte las propiedades organolépticas y texturales deseadas (Bayés-García, 2013), como se detallará a continuación.

\section{La Manteca de Cacao y su Complejo Polimorfismo}

Cualquier tipo de chocolate, ya sea negro, blanco o con leche, contiene tres componentes básicos: manteca de cacao, pasta de cacao y azúcar, los cuales se encuentran en diferentes proporciones en los distintos tipos de producto. Sin embargo, es bien sabido que también puede contener ingredientes adicionales, como leche en polvo (en el chocolate blanco y con leche) o vainilla (en el chocolate blanco), que le aportará sabores y características variados.

La pasta de cacao no es más que el producto resultante del tostado, la molienda y el refinado del cacao en grano, mientras que la manteca de cacao es la fracción grasa del cacao. Así mismo, el cacao, conocido por sus numerosos efectos beneficiosos para la salud, está contenido en la pasta de cacao. En este punto cabe destacar, respecto al etiquetado de una tableta de chocolate, que el tanto por ciento de cacao que se especifica se refiere a la suma del contenido en pasta de cacao y manteca de cacao.

Esta compleja composición del chocolate, se traduce en una sofisticada microestructura, básicamente formada por un entramado de cristales de manteca de cacao donde destacan cristales de azúcar, la cual puede ser caracterizada mediante técnicas microscópicas potentes, como la microscopia electrónica de barrido (ver Figura 1). 


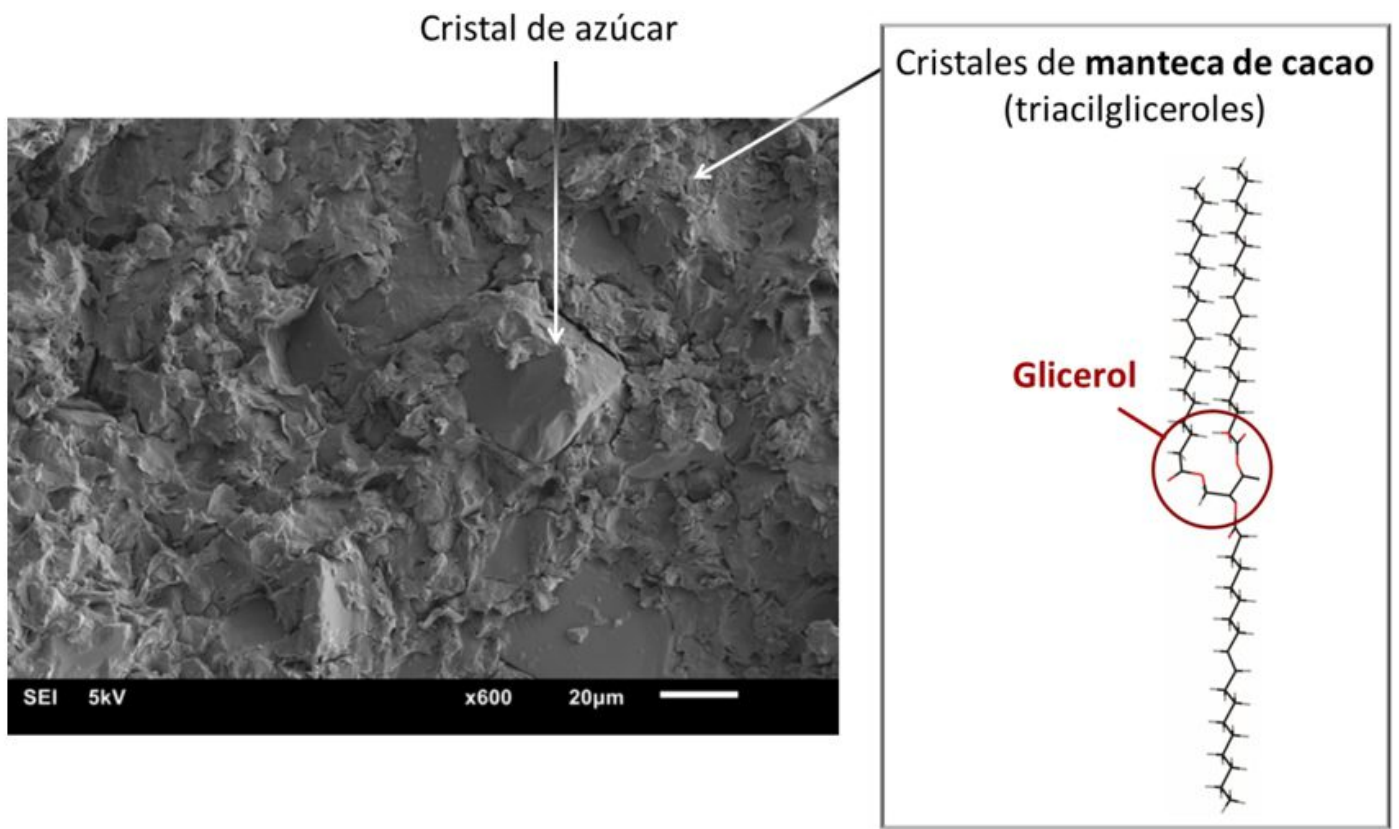

Figura 1. Microestructura del chocolate observada mediante microscopia diferencial de barrido.

De entre todos los componentes del chocolate, el que presenta un complejo comportamiento polimórfico, decisivo para la obtención de las propiedades deseadas del producto final, es la manteca de cacao, la cual está básicamente constituida por triacilgliceroles. Como se muestra en la Figura 1, la molécula de triacilglicerol consiste en un triester de un glicerol con tres ácidos grasos (cadenas). La nomenclatura utilizada para designar e identificar los triacilgliceroles se basa en la secuencia de los ácidos grasos que los constituyen. De este modo, los tres triacilgliceroles básicos de la manteca de cacao son el POP, POS y SOS, constituidos por ácido palmítico (P), oleico (O) y esteárico (S). Estos triacilgliceroles presentan un comportamiento polimórfico altamente complejo, basado en la existencia de hasta siete formas polimórficas diferentes, para cada uno de ellos, las cuales son muy difíciles de aislar del resto puesto que se encuentran coexistiendo con otras formas en la mayoría de condiciones experimentales. Cabe añadir que las técnicas que permiten identificarlas, tales como las técnicas espectroscópicas de Infrarrojo y Raman o la difracción de rayos $X$, aportan unos patrones muy parecidos para las distintas formas, de modo que su identificación no es siempre evidente, hecho que obliga a acudir a técnicas todavía más potentes, como la difracción de rayos $\mathrm{X}$ con radiación sincrotrón.

Cuando los triacilgliceroles POP, POS y SOS se encuentran mezclados en la manteca de cacao, ésta exhibe un comportamiento polimórfico basado en la presencia de seis formas: formas I a VI, en orden creciente de estabilidad. La característica principal que permite definir el polimorfo deseado para que el chocolate presente las propiedades esperadas por el consumidor es su punto de fusión. Como se muestra en la Figura 2(a), cada forma presenta un punto de fusión distinto y, de todas ellas, la que es promocionada por la industria chocolatera es la forma V. En el proceso de fabricación del chocolate, la manteca de cacao debe estar presente en su forma $V$, puesto que funde a una temperatura de $32-33^{\circ} \mathrm{C}$, que coincide con la temperatura de nuestra boca. De este modo, en introducir una pieza de chocolate en nuestra boca, ésta fundirá enseguida, confiriéndonos una sensación refrescante. Esta temperatura de fusión también es adecuada por ser superior a la temperatura de nuestros dedos (de unos $28^{\circ} \mathrm{C}$ aproximadamente), de modo que podemos coger la pieza de chocolate sin que funda antes de degustarla. 
(a)

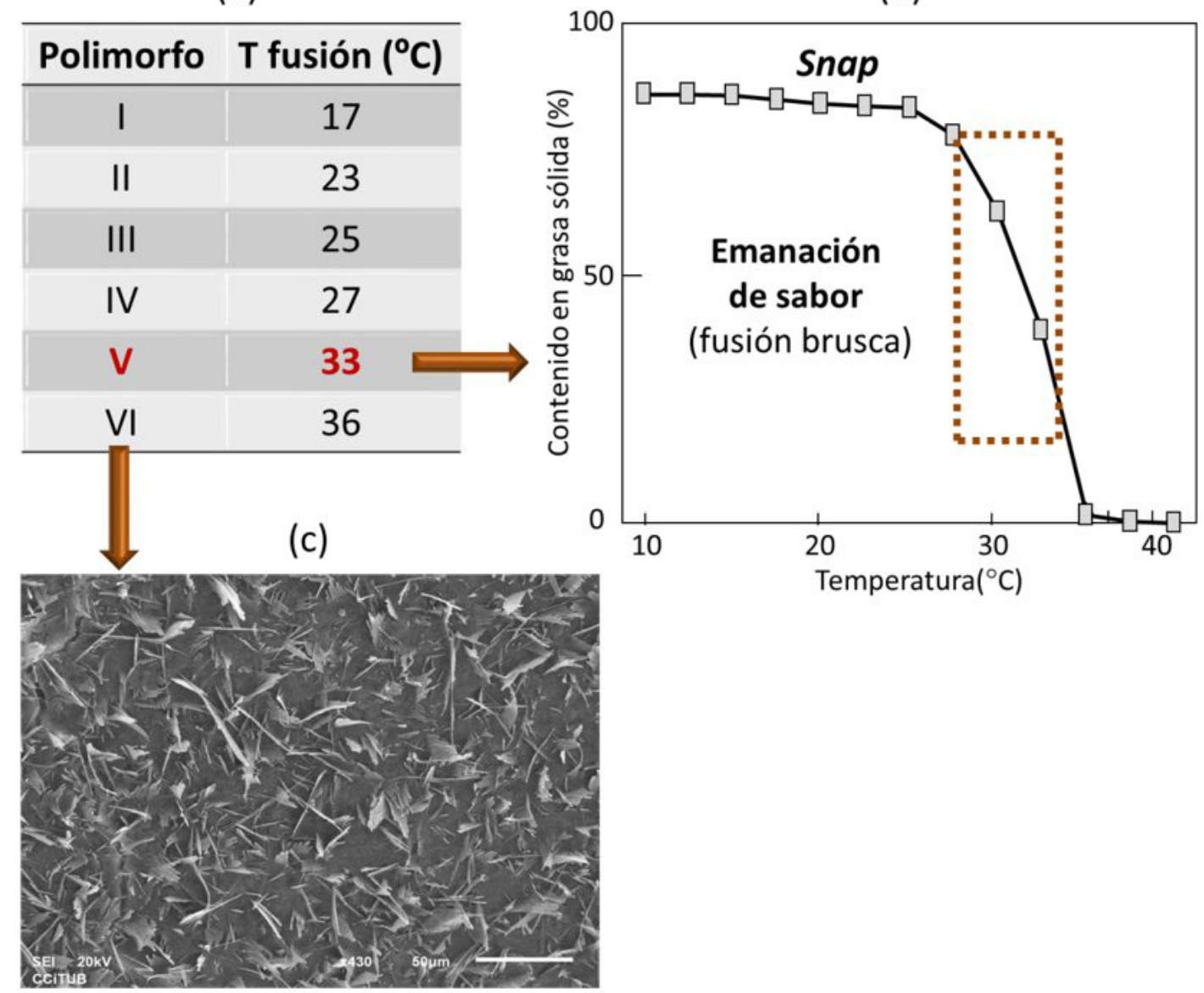

(b)

Figura 2. (a) Temperatura de fusión de los seis polimorfos de la manteca de cacao. (b) Perfil de fusión de la forma V. (c) Morfología de los cristales de forma VI.

Además, esta forma $\mathrm{V}$ presenta un perfil de fusión muy especial y característico. En la Figura $2 \mathrm{~b}$ se muestra la correspondiente evolución de su contenido de grasa sólida en función de la temperatura. Se observa que el contenido en grasa sólida prácticamente no disminuye hasta la temperatura de fusión, manteniendo su carácter crujiente, su snap. En aumentar la temperatura hasta su temperatura de fusión, el contenido en grasa sólida disminuye de manera brusca, momento en que tiene lugar la potente emanación del sabor.

Para conseguir que la manteca de cacao esté cristalizada en forma V, los chocolateros llevan a cabo el conocido proceso de temperado. Este proceso consiste en aplicar, mediante agitación constante, un programa térmico muy concreto al chocolate antes de su moldeado. Siguiendo la tradición, el chocolatero artesanal sigue realizando el temperado del chocolate ayudándose de una pieza de mármol, mientras que a escala industrial se lleva a cabo en máquinas atemperadoras. Dicho tratamiento térmico se constituye de diferentes etapas: (i) Calentamiento del chocolate hasta una temperatura de $45^{\circ} \mathrm{C}$ o superior: fusión de la manteca de cacao); (ii) Enfriamiento hasta unos $27^{\circ} \mathrm{C}$ : formación de núcleos de forma IV; (iii) Calentamiento hasta $32^{\circ} \mathrm{C}$ : transformación polimórfica de la forma IV a forma V; (iv) Etapas de retención y maduración: nucleación completa de la forma $\mathrm{V}$ y crecimiento de los núcleos cristalinos. Mediante el proceso de temperado, se consigue estabilizar la forma $V$ de la manteca de cacao, que confiere al chocolate una textura fina y crujiente, aspecto brillante, y temperatura y perfil de fusión adecuados.

Con la finalidad que dichas propiedades se mantengan en el tiempo durante la vida útil del producto, hay que procurar que la forma $V$ de la manteca de cacao sea estable y no transite a otras formas polimórficas indeseadas. Sin embargo, cuando el chocolate se almacena bajo condiciones de temperatura no adecuadas (ej. temperaturas superiores a los $25^{\circ} \mathrm{C}$ o fluctuaciones de temperatura), tiene lugar el fenómeno denominado Fat Bloom (emanación de grasa) macroscópicamente observable e identificable por la presencia de una capa blanca en la superficie del chocolate. Debido a las temperaturas elevadas, se produce una proporción demasiado elevada de manteca de cacao líquida en la microestructura del chocolate, una fracción de la cual ascenderá 
por capilaridad entre los intersticios y canales creados por cristales de azúcar y manteca de cacao, partículas de cacao, etc. presentes en dicha microestructura (proceso de migración). Al enfriarse este líquido que ha migrado hasta la superficie del chocolate, cristaliza en la forma VI, con una temperatura de fusión más elevada que la forma $\mathrm{V}$, y constituida por cristales de morfología claramente acicular, como muestra la imagen de microscopia electrónica de barrido de la Figura 2(c). Desde el punto de vista organoléptico, la aparición de forma VI supondrá una fusión no inmediata de la manteca de cacao y una textura áspera y terrosa, debido al tamaño considerable de los cristales, por encima del umbral de detección de nuestras papilas gustativas.

Existe una amplia comunidad científica internacional dedicada, en parte, a la búsqueda de una solución para evitar el fenómeno del Fat Bloom, el cual significa uno de los quebraderos de cabeza más tormentosos para los chocolateros. Todavía no se ha encontrado una solución evidente que pueda evitarlo completamente, aunque ya existen diseños de aditivos que permiten retardarlo (Delbaere et al., 2016). Lo que está claro es que la ejecución de un buen proceso de temperado del chocolate, que le otorgue una microestructura robusta y compacta y el cuidado de la temperatura de almacenamiento son aspectos cruciales para evitar la aparición de Fat Bloom. De todos modos, no hay que olvidar que la forma polimórfica VI es termodinámicamente más estable que la $\mathrm{V}$, de modo que, con el tiempo, el sistema tenderá inevitablemente a transformarse en forma VI.

\section{Nuevas Texturas de Chocolate}

El estudio y caracterización de los cristales de manteca de cacao y la influencia de ciertos factores externos sobre su comportamiento cristalino ha llevado a la caracterización y explicación de los fenómenos físico-químicos que rigen la formación y propiedades de texturas desarrolladas de modo tradicional a lo largo de los años. A mediados del siglo XX, ya se usaba la técnica de pintado de piezas de chocolate mediante procesos de pulverización con pistola. En 1963, en la Pastelería Mora de Barcelona, el maestro chocolatero Joan Giner olvidó una mona de chocolate en la nevera durante una sesión de pintado. Sin esperar que la pieza recuperara la temperatura ambiente, se atrevió a pintarla y, con ello, desarrolló el Efecto Terciopelo (ver Figura 3a). Este efecto consiste en una capa delgada, mate $y$, a su vez, rugosa, sobre la superficie del chocolate, que macroscópicamente recuerda al terciopelo y que, en degustarla, confiere una sensación más fina y refrescante que la forma $\mathrm{V}$ del chocolate obtenida por el proceso de temperado convencional. Esta técnica ha sido aplicada desde su desarrollo, pero no ha sido hasta hace muy poco que se han conocido los mecanismos precisos de su formación (Bayés-García et al., 2015).

Técnicas microscópicas, como la microscopia electrónica de barrido, la microscopia confocal interferométrica y la microscopia de fuerzas atómicas permitieron caracterizar la morfología, el perfil y la rugosidad de los agregados esféricos que constituyen el Efecto Terciopelo, formados debido al rápido enfriamiento sufrido por las gotas de chocolate pulverizadas en entrar en contacto con la superficie fría de chocolate. La microscopia electrónica de barrido confirmó la típica microestructura del chocolate en el interior de dichas partículas esféricas, basada en un entramado de cristales de manteca de cacao con cristales de azúcar incrustados (ver Figura 3b). 
(a)

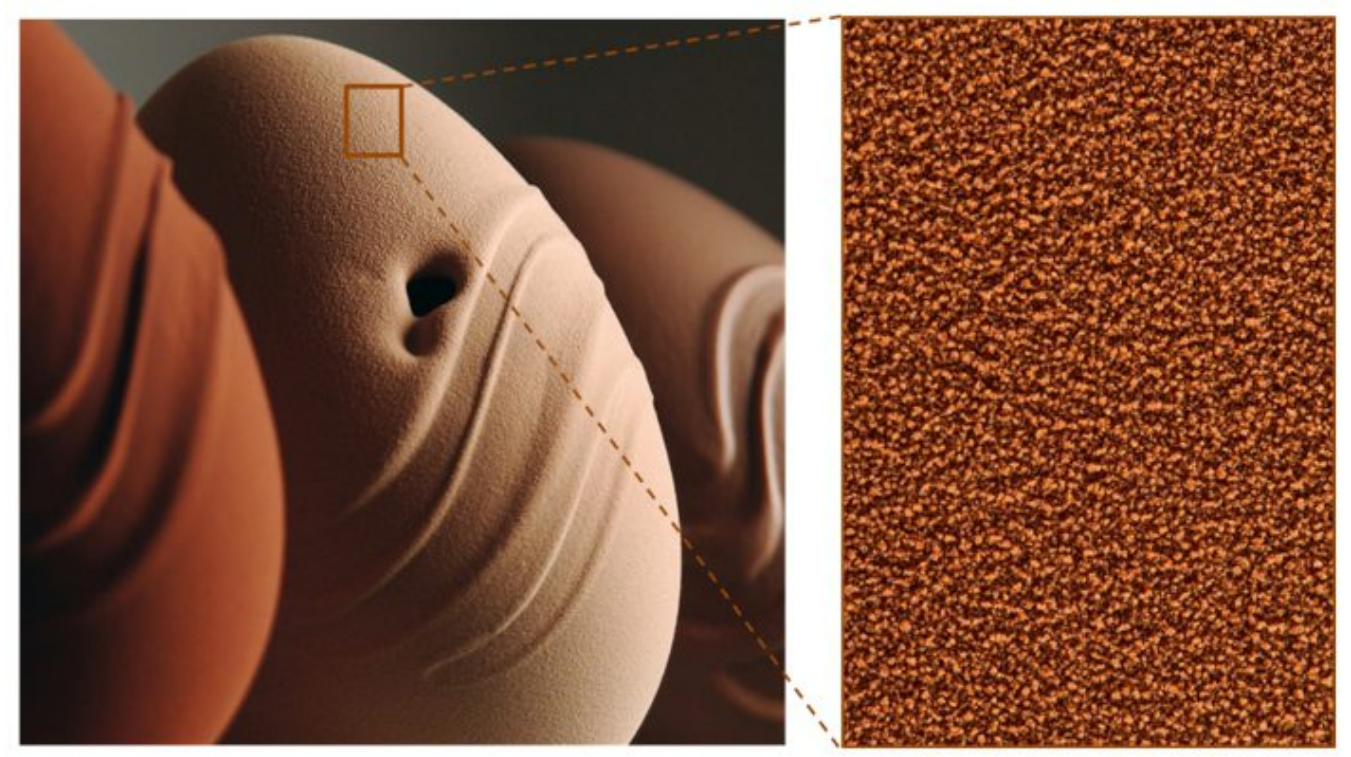

(b)
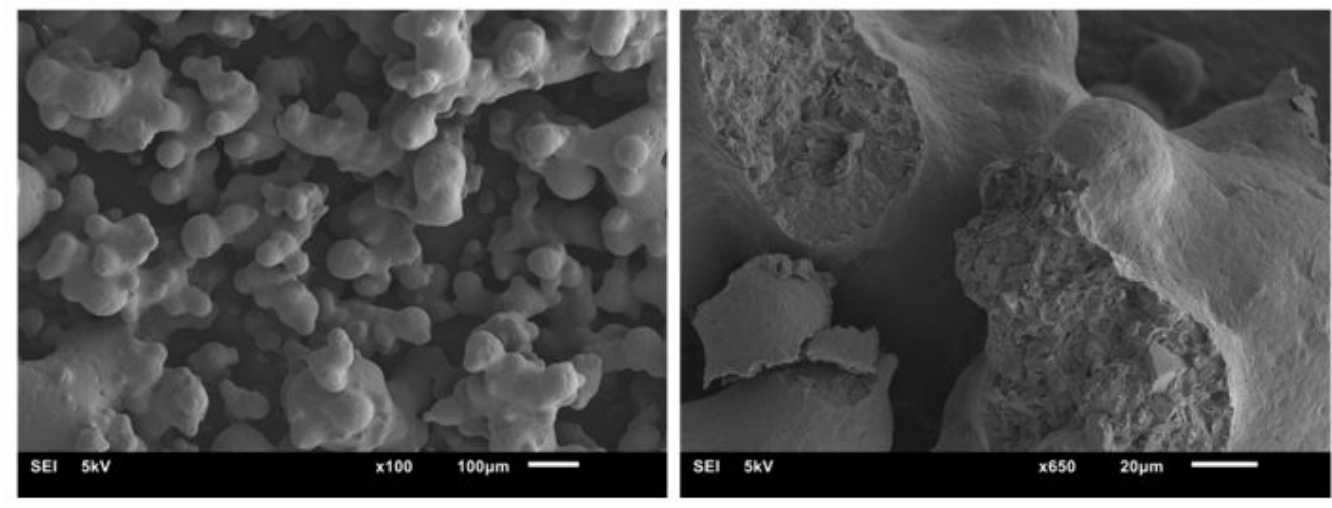

Figura 3. Efecto Terciopelo (a) Imágenes macroscópicas (cedidas por el maestro chocolatero Enric Rovira). (b) Imágenes de microscopia electrónica de barrido.

Organolépticamente, el efecto terciopelo confiere una fusión más rápida en boca que el chocolate normalmente temperado, hecho que se confirmó con técnicas calorimétricas, como la calorimetría diferencial de barrido, que demostró una temperatura de fusión unos dos grados inferior a la del chocolate convencional. Por otro lado, la difracción de rayos $\mathrm{X}$ mostró difractogramas similares, revelando la presencia única de forma $V$ en ambos casos. Sin embargo, los picos de difracción correspondientes a la muestra de Efecto Terciopelo resultaron ser más anchos que los del chocolate convencional, hecho que normalmente se asocia a dominios cristalinos de tamaño inferior. Dichos resultados fueron confirmados con análisis mediante radiación sincrotrón.

Para comprender el mecanismo mediante el cual el Efecto Terciopelo contenía cristales de manteca de cacao de menor tamaño que el chocolate convencional, se monitorizó sistemáticamente el proceso de formación de la forma V. Los resultados mostraron que la baja temperatura del chocolate sobre la que se llevaba a cabo la pulverización provocaba una cristalización inicial de la manteca de cacao en forma I, forma metaestable caracterizada por un pequeño tamaño cristalino. A medida que el sistema evolucionaba a temperatura ambiente, tuvo lugar una secuencia de transformación polimórfica: forma I $\rightarrow$ forma II $\rightarrow$ forma V. De este modo, los pequeños cristales de forma I evolucionaron a forma $V$ (a través de la forma II), manteniéndose el pequeño tamaño cristalino, el cual resultó ser el responsable del bajo punto de fusión del Efecto Terciopelo, si se compara con el del chocolate normalmente temperado.

De este modo, el polimorfismo, propiedad que presentan muchos minerales y otros sólidos cristalinos de cristalizar en estructuras diferentes, según las condiciones de temperatura y presión entre otros, también ejerce un rol decisivo en los productos alimentarios, campo donde también debe ser estrictamente analizado. El uso de ciertos factores externos, como la aplicación de tratamientos térmicos específicos, permiten obtener las propiedades deseadas del producto final, 
para las cuales es necesario el conocimiento, la monitorización y el control de su complejo comportamiento.

\section{Bibliografía}

Larsson, K.; Quinn, P.; Sato, K; Tiberg, F. (Eds.) Lipids: Structure, Physical Properties and Functionality. The Oily Press, Bridgwater, 2006.

Bayés-García, L. Sorprenentment, mengem cristalls!. Ciència i Xocolata, Edicions Universitat de Barcelona, Barcelona, 2013.

Bayés-García, L.; Calvet, T.; Cuevas-Diarte, M. A.; Rovira, E.; Ueno, S.; Sato, K. New Textures of Chocolate are Formed by Polymorphic Crystallization and Template Effects: Velvet Chocolate. Crystal Growth \& Design, 2015, 15, 4045-4054.

Bayés-García, L.; Tres, A.; Vichi, S.; Calvet, T.; Cuevas-Diarte, M. A.; Codony, R.; Boatella, J.; Caixach, J.; Ueno, S.; Guardiola, F. Authentication of Iberian dry-cured ham: New approaches by polymorphic fingerprint and ultrahigh resolution mass spectrometry. Food Control, 2016, 60, 370377.

Delbaere, C.; Van de Walle, D.; Depypere, F.; Gellynck, X.; Dewettinck, K. Relationship between chocolate microstructure, oil migration, and fat bloom in filled chocolates. European Journal of Lipid Science and Technology, 2016, 118, 1800-1826.

Tierra y Tecnología no 49 | Autores: Laura Bayés-García, Miquel Àngel Cuevas-Diarte, Teresa Calvet. Secció de Cristal·lografia, Mineralogia i Dipòsits Minerals, Facultat de Ciències de la Terra, Universitat de Barcelona | http://dx.doi.org/10.21028/lbg.2017.01.09

Laura Bayés-García | Profesora Lectora del Departamento de Mineralogía, Petrología y Geología Aplicada de la Universitat de Barcelona (UB). Doctora Internacional por la UB (2013), Máster en Cristalografía y Cristalización (Universidad Internacional Menéndez Pelayo, 2009), Licenciada en Ciencia y Tecnología de los Alimentos (UB, 2008, donde le concedieron el Premio Extraordinario de Licenciatura) y Licenciada en Química (UB, 2006). Recientemente (2014) ganó el Premio Xavier Solans por el mejor trabajo en Cristalografía y Crecimiento Cristalino realizado por un investigador joven en España. La investigación que lleva a cabo en el Grupo de Aleaciones Moleculares de la Sección de Cristalografía, Mineralogía y Depósitos Minerales de la UB se basa en la caracterización polimórfica de grasas y aceites alimentarios, con el objetivo de mejorar las características físicoquímicas del producto final o la detección de fraudes alimentarios.

Teresa Calvet Pallas I Doctora en Geología (1990) y profesora Titular de la UB (1992), su investigación está centrada en la caracterización cristalográfica y termodinámica del polimorfismo y miscibilidad en estado sólido de diferentes materiales. En los últimos años esta actividad se ha centrado en la caracterización cristalográfica de productos alimenticios (grasas y aceites), desde los componentes (triacilgliceroles) hasta el producto final (chocolate, aceites vegetales, jamón Ibérico) con el objetivo de mejorar la textura, alargar la vida útil o la detección de fraudes alimentarios. Colabora con otros grupos de investigación internacionales, así como de la Universidad de Barcelona y la Universidad Autónoma de Barcelona en la resolución de estructuras a partir de datos de difracción con monocristal de rayos X. Es coautora de 2 libros y de unas 150 publicaciones indexadas en el SCI; ha dirigido 6 Tesis Doctorales y actualmente es coordinadora del Grupo de Aleaciones Moleculares y del Grupo de Investigación Reconocido de Cristalografía.

Miquel Àngel Cuevas-Diarte | Es catedrático emérito de Cristal-lografia y Mineralogia de la Universitat de Barcelona. Especialista en polimorfismo y miscibilidad en estado sólido, actualmente trabaja en Cristal-lografia de componentes alimentarios. Autor de un centenar largo de publicaciones, y coautor de dos patentes, recibió la mención especial del Premio Ciudad de Barcelona en investigación tecnológica en 1998. Ha impartido docencia sobre diferentes aspectos 
de la Cristal·lografia, así como conferencies, semanarios y talleres de divulgación. Ha sido el coordinador de las cinco primeras ediciones del concurso de cristalización en la escuela en Cataluña. Es autor, coautor y editor de Problemes de Cristal-lografia (2006), Simetria. Un passeig interdisciplinari (2015), y Simetries per a tothom. Un recorregut ple de sorpreses per l'art i la ciència (2016) publicados por Publicacions i Edicions de la Universitat de Barcelona.

\section{Redacción}

http://www.icog.es

Tierra y Tecnología es una publicación del Ilustre Colegio Oficial de Geólogos (ICOG). El ICOG es una institución sin finalidad lucrativa creada para la defensa y apoyo de los intereses de los Geólogos, creada por Ley 73/1978 de 26 de Diciembre, es una corporación de Derecho Público, amparada por Ley y reconocida por el Estado, con personalidad jurídica propia y capacidad plena para el ejercicio de sus funciones y cumplimiento de sus fines.

\section{f $\quad$ + in}

